International Journal of Pure and Applied Mathematics

Volume 95 No. 2 2014, 297-308

ISSN: 1311-8080 (printed version); ISSN: 1314-3395 (on-line version)

url: http://www.ijpam.eu

doi: http://dx.doi.org/10.12732/ijpam.v95i2.13

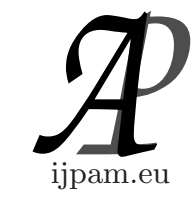

\title{
EXISTENCE OF FUZZY SOLUTIONS FOR IMPULSIVE SEMILINEAR DIFFERENTIAL EQUATIONS WITH NONLOCAL CONDITION
}

\author{
S. Vengataasalam ${ }^{1}$, R. Ramesh ${ }^{2} \S$ \\ ${ }^{1}$ Department of Mathematics \\ Kongu Engineering College \\ Erode, 638 052, Tamil Nadu, INDIA \\ ${ }^{2}$ Department of Mathematics \\ K.S. Rangasamy College of Technology \\ Tiruchengode, 637 215, Tamil Nadu, INDIA
}

\begin{abstract}
In this paper,the work is focussed on the study of fuzzy impulsive semilinear differential equations with nonlocal condition.The results are obtained by using the fixed point principles.
\end{abstract}

AMS Subject Classification: 45G10, 34A07, 34K45

Key Words: impulsive fuzzy differential equation, Nonlocal condition, fixed point theorem, mean-square calculas, semigroup

\section{Introduction}

Zadeh [18] introduced the concept of fuzzy sets.The topic of fuzzy differential equations has been rapidly growing in recent years. They play a important role both in theory and application, for example, in population models, in engineering, in chaotic systems and in modeling hydraulics.A large class of physically important problems is described by fuzzy differential equations [8],[15],[17].

Byszewski [3] investigated the existence and uniqueness of mild, strong, and classical solutions of a nonlocal cauchy problem for a semilinear evolu-

Received: May 7, 2014

(c) 2014 Academic Publications, Ltd.

${ }^{\S}$ Correspondence author url: www.acadpubl.eu 
tion equation.For the monographs of the theory of impulsive diffferential equations, we can refer the books of Bainov and Simenov[1],Lakshmikantham et.al [10],Samoilenko and Perestyuk [14] and in papers [9],[11],[2] where numerous properties of their solutions are studied.

Kaleva [7] discussed the properties of differentiable fuzzy set-valued mappings by means of the concept of H-differentiability.Feng [4] studied the existence and uniqueness of a solution, the continuity of the solution with respect to the initial value and the stability of fuzzy stochastic differential equations.Tung [16] discussed the existence and some comparison results on solutions of fuzzy control stochastic differential systems and investigated the continuous dependence of solutions.Jeong [6] studied fuzzy differential equations with nonlocal condition.Ramesh [13] studied the fuzzy solutions for impulsive delay integrodifferential equations with nonlocal condition.

Here in this paper,we prove the existence and uniqueness theorem of a solution to the following nonlocal fuzzy impulsive differential equation

$$
\begin{gathered}
x^{\prime}(t)=A x(t)+f(t, x(t)), t \in I=[0, a], \\
x(0)=g\left(t_{1}, t_{2}, \ldots, t_{p}, x(.)\right)+x_{0}, \\
\Delta x\left(t_{k}\right)=I_{k}\left(x\left(t_{k}\right)\right), k=1,2 \ldots, m
\end{gathered}
$$

where $A:[0, T] \rightarrow E_{N}$ is a fuzzy coeffiecient,0 $<t_{1}<t_{2}<\ldots<t_{p} \leq$ a, $f: I \times L_{2} \rightarrow L_{2}$ is mean square continuous fuzzy mapping with respect to t which satisfies a generalized Lipschitz condition, $g: I^{p} \times L_{2} \rightarrow L_{2}$ satisfies a generalized Lipschitz condition, and $x_{0} \in L_{2}$.Hence (from [5])

$$
L_{2}=\left\{X \mid \mathrm{X} \text { is a fuzzy random variable with } E\left(\|X\|^{2}\right)<\infty\right\}
$$

$\Delta x\left(t_{k}\right)=x\left(t_{k}^{+}\right)-x\left(t_{k}^{-}\right)$, where $x\left(t_{k}^{-}\right)$and $x\left(t_{k}^{+}\right)$represent the left and right limits of $x(t)$ at $t=t_{k}$ respectively. The symbol $g\left(t_{1}, t_{2}, \ldots, t_{p}, x().\right)$ is used in the sense that in the place of '?' we can substitute only elements of the set $\left\{t_{1}, t_{2}, \ldots, t_{p}\right\}$. For example, $g\left(t_{1}, t_{2}, \ldots, t_{p}, x().\right)$ can be defined by the formula

$$
g\left(t_{1}, t_{2}, \ldots, t_{p}, x(.)\right)=c_{1} x\left(t_{1}\right)+c_{2} x\left(t_{2}\right)+\ldots+c_{p} x\left(t_{p}\right)
$$

where $c_{i}(i=1,2, \ldots, p)$ are given constants.

The outlay of the paper is as follows : In section 2 we give some basic definition for our study. In Section 3 we prove the main theorem on the existence of fuzzy solutions. 


\section{Preliminaries}

The symbol $P_{C}\left(R^{n}\right)$ denotes the family of all nonempty compact convex subsets of $R^{n}$. Define the addition and scalar multiplication in $P_{C}\left(R^{n}\right)$ as usual. Denote $E^{n}=\left\{u: R^{n} \rightarrow[0,1], u\right.$ satisfies $(i)-(i v)$ below $\}$, where

(i) $u$ is normal, i.e., there exists an $x_{0} \in R^{n}$ such that $u\left(x_{0}\right)=1$;

(ii) $u$ is fuzzy convex, i.e., $u(r x+(1-r) y) \geq \min (u(x), u(y))$, $x, y \in R^{n}, r \in[0,1] ;$

(iii) $u$ is upper semicontinuous;

(iv) $[u]^{0}=\left\{x \in R^{n} \mid u(x)>0\right\}$ is compact.

Let $u, v \in E^{n}$, and set

$$
D(u, v)=\sup _{0 \leq r \leq 1} d\left([u]^{r},[v]^{r}\right),
$$

where $[u]^{r}=\left\{x \in R^{n} \mid u(x) \geq r\right\}, 0<r \leq 1$, is the $r$-level set of $u, d$ is the hausdorff metric defined in $P_{C}\left(R^{n}\right)$. i.e.,

$$
d(A, B)=\max \left(\sup _{a \in A} \inf _{b \in B}|a-b|, \sup _{b \in B} \inf _{a \in A}|a-b|\right),
$$

for all $A, B \in P_{C}\left(R^{n}\right)$, where |.| denotes the usual Euclidean norm in $R^{n} .\left(E^{n}, D\right)$ is a complete metric space(see [12]).

Let $(\Omega, A, P)$ be a complete probability space. A fuzzy random variable is a Borel measurable function $X:(\Omega, A) \rightarrow\left(E^{n}, D\right)$. Let

$L_{2}(\Omega, A, P)=\left\{X \mid X\right.$ is a fuzzy random variable with $\left.\int_{\Omega} D(X, \hat{0})^{2} d P(w)<\infty\right\}$.

Two fuzzy random variables $\mathrm{X}$ and $\mathrm{Y}$ are called equivalent if $P(X \neq Y)=0$. The all equivalent element in $L_{2}$ are identified. Define

$$
\varphi(X, Y)=\left(\int_{\Omega}(D(X, Y))^{2} d P\right)^{\frac{1}{2}}, X, Y \in L_{2} .
$$

The norm $\|X\|_{2}$ of an element $X \in L_{2}$ is defined by

$$
\|X\|_{2}=\varphi(X, \hat{0})=\left(\int_{\Omega}(D(X, \hat{0}))^{2} d P\right)^{\frac{1}{2}} .
$$


Then $\left(L_{2}, \varphi\right)$ is a complete metric space [4] and $\varphi$ satisfies that $\varphi(X+Z, Y+Z)=\varphi(X, Y), \varphi(\lambda X, \lambda Y)=|\lambda| \varphi(X, Y), \varphi(\lambda X, k X) \leq|\lambda-k|\|X\|_{2}$ for any $X, Y, Z \in L_{2}$ and $\lambda, k \in R$.

\section{Fuzzy solutions}

In this section, We consider the following nonlocal fuzzy impulsive differential equation:

$$
\begin{gathered}
x^{\prime}(t)=A x(t)+f(t, x(t)), t \in I=[0, a], \\
x(0)=g\left(t_{1}, t_{2}, \ldots, t_{p}, x(.)\right)+x_{0}, \\
\Delta x\left(t_{k}\right)=I_{k}\left(x\left(t_{k}\right)\right), k=1,2 \ldots, m
\end{gathered}
$$

where $A:[0, T] \rightarrow E_{N}$ is a fuzzy coeffiecient, $0<t_{1}<t_{2}<\ldots<t_{p} \leq$ $a, f: I \times L_{2} \rightarrow L_{2}$ is mean square continuous fuzzy mapping with respect to t which satisfies a generalized Lipschitz condition, $g: I^{p} \times L_{2} \rightarrow L_{2}$ satisfies a generalized Lipschitz condition and $x_{0} \in L_{2}$ and $\Delta x\left(t_{k}\right)=x\left(t_{k}^{+}\right)-x\left(t_{k}^{-}\right)$, where $x\left(t_{k}^{-}\right)$and $x\left(t_{k}^{+}\right)$represent the left and right limits of $x(t)$ at $t=t_{k}$ respectively.

Theorem 1. Assume the following

(H1) Let $f: I \times L_{2} \rightarrow L_{2}$ be mean square continuous with respect to $t$ and there exists constants $L$ such that

$$
H_{d}(f(t, x), f(t, y)) \leq L H_{d}(x, y)
$$

(H2) Let $g: I^{p} \times L_{2} \rightarrow L_{2}$ satisfies a generalized Lipschitz condition and there exists a constant $K$ such that

$$
H_{d}\left(g\left(t_{1}, \ldots, t_{p}, x(.)\right), g\left(t_{1}, \ldots, t_{p}, y(.)\right)\right) \leq K H_{d}(x, y), \forall t \in I, x, y \in L_{2} .
$$

(H3) Let $\xi=\min \left\{a, \frac{b-N}{M}, \frac{1-K}{L}\right\}$ where $M, N$ are defined as,

$$
H_{d}(f(t, x), \hat{0}) \leq M, H_{d}\left(g\left(t_{1}, \ldots, t_{p}, x(.)\right), \hat{0}\right) \leq N
$$

(H4) Let $S(t)$ is a fuzzy number such that $|S(t)| \leq c, \forall t \in I$ 
(H5) There exists a constant $\beta_{k}$ and $\chi$ such that

$$
\begin{gathered}
H_{d}\left(I_{k}\left(x\left(t_{k}\right)\right), I_{k}\left(y\left(t_{k}\right)\right)\right) \leq \beta_{k} \text { and } \\
H_{d}\left(\sum_{0<t<t_{k}} I_{k}\left(x\left(t_{k}\right)\right), \hat{0}\right) \leq \chi
\end{gathered}
$$

Then the equation (1)-(3) has a unique solution on the interval $[0, \xi]$.

Proof. Let $B=\left\{x \in L_{2} \mid H\left(x, x_{0}\right) \leq b\right\}$ be the space of mean square continuous fuzzy mappings with

$$
H(x, y)=\sup _{0 \leq t \leq \xi} H_{d}(x(t), y(t))
$$

and $\mathrm{b}$ a positive number. Define a mapping $G: B \rightarrow B$ by

$$
\begin{aligned}
G x(t)=S(t) x_{0}+S(t) g\left(t_{1}, \ldots, t_{p}, x(.)\right)+\int_{0}^{t} S(t & -s) f(s, x(s)) d s \\
& +\sum_{0<t<t_{k}} S\left(t-t_{k}\right) I_{k}\left(x\left(t_{k}\right)\right) .
\end{aligned}
$$

First of all,we show that $G$ is mean square continuous and $H\left(G x, x_{0}\right) \leq b$. Since $\mathrm{f}$ is mean square continuous, we have

$$
\begin{aligned}
H_{d}(G x & (t+h), G x(t)) \\
= & H_{d}\left(S(t+h) x_{0}+S(t+h) g\left(t_{1}, \ldots, t_{p}, x(.)\right)+\int_{0}^{t+h} S(t+h-s) f(s, x(s)) d s\right. \\
& +\sum_{0<t<t_{k}} S\left(t+h-t_{k}\right) I_{k}\left(x\left(t_{k}\right)\right), S(t) x_{0}+S(t) g\left(t_{1}, \ldots, t_{p}, x(.)\right) \\
& \left.+\int_{0}^{t} S(t-s) f(s, x(s)) d s+\sum_{0<t<t_{k}} S\left(t-t_{k}\right) I_{k}\left(x\left(t_{k}\right)\right)\right) \\
\leq & H_{d}\left(S(t+h) x_{0}, S(t) x_{0}\right)+H_{d}\left(S(t+h) g\left(t_{1}, \ldots, t_{p}, x(.)\right), S(t) g\left(t_{1}, \ldots, t_{p}, x(.)\right)\right) \\
& +H_{d}\left(\int_{0}^{t+h} S(t+h-s) f(s, x(s)) d s, \int_{0}^{t} S(t-s) f(s, x(s) d s)\right) \\
& +H_{d}\left(\sum_{0<t<t_{k}} S\left(t+h-t_{k}\right) I_{k}\left(x\left(t_{k}\right)\right), \sum_{0<t<t_{k}} S\left(t-t_{k}\right) I_{k}\left(x\left(t_{k}\right)\right)\right) \\
\leq & c\left(\int_{t}^{t+h} H_{d}(f(s, x(s)), \hat{0}) d s \quad\right.
\end{aligned}
$$




$$
\leq \operatorname{ch} M \rightarrow 0 \quad(\text { as } \quad h \rightarrow 0)
$$

That is, the map $G$ is mean square continuous on I. Furthermore,

$$
\begin{aligned}
H_{d}\left(G x(t), x_{0}\right) \leq & H_{d}\left(S(t) g\left(t_{1}, \ldots, t_{p}, x(.)\right), \hat{0}\right)+H_{d}\left(\int_{0}^{t} S(t-s) f(s, x(s)) d s, \hat{0}\right) \\
& +H_{d}\left(\sum_{0<t<t_{k}} S\left(t-t_{k}\right) I_{k}\left(x\left(t_{k}\right)\right), \hat{0}\right) \\
\leq & c(N+M t+\chi),
\end{aligned}
$$

and so

$$
\begin{aligned}
H\left(G x, x_{0}\right) & =\sup _{0 \leq t \leq \xi} H_{d}\left(G x(t), x_{0}\right) \\
& \leq c(N+M \xi+\chi) \\
& \leq b
\end{aligned}
$$

Since $\left(L_{2}, H_{d}\right)$ is a complete metric space, a standard proof applies to show that

$$
C\left([0, \xi], L_{2}\right)=\left\{x:[0, \xi] \rightarrow L_{2} \mid \mathrm{x}(\mathrm{t}) \text { is mean square continuous }\right\}
$$

is complete. Now we show that B is a closed subset of $C\left([0, \xi], L_{2}\right)$. Let $\left\{x_{n}\right\}$ be a sequence in B such that $x_{n} \rightarrow x \in C\left([0, \xi], L_{2}\right)$ as $n \rightarrow \infty$.

Then

$$
\begin{aligned}
H_{d}\left(x(t), x_{0}\right) & \leq H_{d}\left(x(t), x_{n}(t)\right)+H_{d}\left(x_{n}(t), x_{0}\right) . \\
H\left(x, x_{0}\right) & =\sup _{0 \leq t \leq \xi} H_{d}\left(x(t), x_{0}\right) \\
& \leq H\left(x, x_{n}\right)+H\left(x_{n}, x_{0}\right) \\
& \leq \varepsilon+b
\end{aligned}
$$

for sufficiently large $n$ and arbitrary $\varepsilon>0$. So $x \in B$. This implies that $\mathrm{B}$ is a closed subset of $C\left([0, \xi], L_{2}\right)$. Therefore $\mathrm{B}$ is a complete metric space. Next,we will show that $G$ is a contraction mapping. For $x, y \in B$,

$$
\begin{aligned}
H_{d}(G x(t), G y(t)) \leq & H_{d}\left(S(t) x_{0}, S(t) y_{0}\right) \\
& +H_{d}\left(S(t) g\left(t_{1}, \ldots, t_{p}, x(.)\right), S(t) g\left(t_{1}, \ldots, t_{p}, y(.)\right)\right) \\
& +H_{d}\left(\int_{0}^{t} S(t-s) f(s, x(s)) d s, \int_{0}^{t} S(t-s) f(s, y(s)) d s\right)
\end{aligned}
$$




$$
\begin{aligned}
& +H_{d}\left(\sum_{0<t<t_{k}} S\left(t-t_{k}\right) I_{k}\left(x\left(t_{k}\right)\right), \sum_{0<t<t_{k}} S\left(t-t_{k}\right) I_{k}\left(y\left(t_{k}\right)\right)\right) \\
\leq & c K H_{d}(x, y) \\
& +c \int_{0}^{t} L H_{d}(x(s), y(s)) d s+c H_{d}\left(I_{k}\left(x\left(t_{k}\right)\right), I_{k}\left(y\left(t_{k}\right)\right)\right) .
\end{aligned}
$$

Thus, we obtain

$$
\begin{aligned}
H(G x, G y) \leq & \sup _{0 \leq t \leq \xi}\left\{c K H_{d}(x, y)\right. \\
& +c L \int_{0}^{t} H_{d}(x(s), y(s)) d s+c H_{d}\left(I_{k}\left(x\left(t_{k}\right)\right), I_{k}\left(y\left(t_{k}\right)\right)\right\} . \\
\leq & c\left(K+\xi L+\beta_{k}\right) H(x, y) .
\end{aligned}
$$

since $c\left(K+\xi L+\beta_{k}\right)<1, G$ is a contraction map. Therefore $G$ has a unique fixed point $G x=x \in C\left([0, \xi], E^{n}\right)$, that is

$$
\begin{aligned}
x(t)=S(t) x_{0}+S(t) g\left(t_{1}, \ldots, t_{p}, x(.)\right)+ & \int_{0}^{t} S(t-s) f(s, x(s)) d s \\
& +\sum_{0<t<t_{k}} S\left(t-t_{k}\right) I_{k}\left(x\left(t_{k}\right)\right) .
\end{aligned}
$$

Theorem 2. Suppose that $f, g$ are the same as in theorem 1. Let $x\left(t, x_{0}\right), y\left(t, y_{0}\right)$ be the solutions of Eq.(1)-(3) to $x_{0}, y_{0}$ respectively. Then there exist constants $c_{1}$ and $c_{2}$ such that

(i) $H\left(x\left(., x_{0}\right), y\left(., y_{0}\right) \leq c_{1}\left(H_{d}\left(x_{0}, y_{0}\right)+\beta_{k}\right)\right.$ for any $x_{0}, y_{0} \in L_{2}$,

(ii) $H\left(x\left(., x_{0}\right), \hat{0}\right) \leq c_{2}\left(H_{d}\left(x_{0}, \hat{0}\right)+N+M+\chi\right)$, where

$$
\begin{aligned}
& H_{d}\left(g\left(t_{1}, \ldots, t_{p}, x(.), \hat{0}\right) \leq N, \int_{0}^{t} H_{d}(f(s, \hat{0}), \hat{0}) d s \leq M,\right. \\
& H_{d}\left(\sum_{0<t<t_{k}} I_{k}\left(x\left(t_{k}\right)\right), \hat{0}\right] \leq \chi, H_{d}\left(I_{k}\left(x\left(t_{k}\right)\right), I_{k}\left(y\left(t_{k}\right)\right)\right) \leq \beta_{k} .
\end{aligned}
$$

Proof. (i) For any $t \in[0, \xi]$, we have

$$
\begin{aligned}
& H_{d}\left(x\left(t, x_{0}\right), y\left(t, y_{0}\right)\right) \\
& \leq H_{d}\left(S(t) x_{0}+S(t) g\left(t_{1}, \ldots, t_{p}, x\left(., x_{0}\right)\right)+\int_{0}^{t} S(t-s) f\left(s, x\left(s, x_{0}\right)\right) d s\right.
\end{aligned}
$$




$$
\begin{aligned}
& \quad+\sum_{0<t<t_{k}} S\left(t-t_{k}\right) I_{k}\left(x\left(t_{k}\right)\right), S(t) y_{0}+S(t) g\left(t_{1}, \ldots, t_{p}, y\left(., y_{0}\right)\right) \\
& \left.+\int_{0}^{t} S(t-s) f\left(s, y\left(s, y_{0}\right)\right) d s+\sum_{0<t<t_{k}} S\left(t-t_{k}\right) I_{k}\left(y\left(t_{k}\right)\right)\right) \\
& \leq c H_{d}\left(x_{0}, y_{0}\right)+c K H_{d}\left(x\left(., x_{0}\right), y\left(., y_{0}\right)\right)+c L \int_{0}^{t} H_{d}\left(x\left(s, x_{0}\right), y\left(s, y_{0}\right)\right) d s \\
& +H_{d}\left(\sum_{0<t<t_{k}} S\left(t-t_{k}\right) I_{k}\left(x\left(t_{k}\right)\right), \sum_{0<t<t_{k}} S\left(t-t_{k}\right) I_{k}\left(y\left(t_{k}\right)\right)\right) .
\end{aligned}
$$

From Gronwall's inequality,we get

$$
H_{d}\left(x\left(t, x_{0}\right), y\left(t, y_{0}\right)\right) \leq c\left[H_{d}\left(x_{0}, y_{0}\right)+K H_{d}\left(x\left(., x_{0}\right), y\left(., y_{0}\right)\right)+\beta_{k}\right] \exp L \xi .
$$

Thus we have

$$
H\left(x\left(., x_{0}\right), y\left(., y_{0}\right)\right) \leq c\left[H_{d}\left(x_{0}, y_{0}\right)+K H\left(x\left(., x_{0}\right), y\left(., y_{0}\right)\right)+\beta_{k}\right] \exp L \xi .
$$

i.e.,

$$
(1-c K \exp L \xi) H\left(x\left(., x_{0}\right), y\left(., y_{0}\right)\right) \leq c\left(H_{d}\left(x_{0}, y_{0}\right)+\beta_{k}\right) \exp L \xi
$$

Consequently,we obtain

$$
H\left(x\left(., x_{0}\right), y\left(., y_{0}\right)\right) \leq \frac{c . \exp L \xi}{1-c K \exp L \xi}\left(H_{d}\left(x_{0}, y_{0}\right)+\beta_{k}\right)
$$

Taking $c_{1}=\frac{c \cdot \exp L \xi}{1-c K \exp L \xi}$, we obtain

$$
H\left(x\left(., x_{0}\right), y\left(., y_{0}\right)\right) \leq c_{1}\left(H_{d}\left(x_{0}, y_{0}\right)+\beta_{k}\right) .
$$

(ii) For any $t \in[0, \xi]$,

$$
\begin{aligned}
H_{d}\left(x\left(t, x_{0}\right), \hat{0}\right) \leq & H_{d}\left(S(t) x_{0}, \hat{0}\right)+H_{d}\left(S(t) g\left(t_{1}, \ldots, t_{p}, x\left(., x_{0}\right)\right), \hat{0}\right) \\
& +\int_{0}^{t} H_{d}\left(S(t-s) f\left(s, x\left(s, x_{0}\right)\right), f(s, \hat{0})\right) d s \\
& +\int_{0}^{t} H_{d}(S(t-s)(f(s, \hat{0}), \hat{0}) d s \\
& +H_{d}\left(\sum_{0<t<t_{k}} S\left(t-t_{k}\right) I_{k}\left(x\left(t_{k}\right)\right), \hat{0}\right) \\
\leq & c H_{d}\left(x_{0}, \hat{0}\right)+c H_{d}\left(g\left(t_{1}, \ldots, t_{p}, x\left(., x_{0}\right)\right), \hat{0}\right)
\end{aligned}
$$




$$
\begin{aligned}
& +c L \int_{0}^{t} H_{d}\left(x\left(s, x_{0}\right), \hat{0}\right) d s+c \int_{0}^{t} H_{d}(f(s, \hat{0}), \hat{0}) d s \\
& +c H_{d}\left(\sum_{0<t<t_{k}} I_{k}\left(x\left(t_{k}\right)\right), \hat{0}\right) .
\end{aligned}
$$

From Gronwall's inequality,we get

$$
\begin{aligned}
H_{d}\left(x\left(t, x_{0}\right), \hat{0}\right) \leq c[ & H_{d}\left(x_{0}, \hat{0}\right)+H_{d}\left(g\left(t_{1}, \ldots, t_{p}, x\left(., x_{0}\right)\right), \hat{0}\right) \\
& \left.\quad+\int_{0}^{t} H_{d}(f(s, \hat{0}), \hat{0}) d s+H_{d}\left(\sum_{0<t<t_{k}} I_{k}\left(x\left(t_{k}\right)\right), \hat{0}\right)\right] \exp L t \\
\leq & c\left(H_{d}\left(x_{0}, \hat{0}\right)+N+M+\chi\right) \exp L \xi .
\end{aligned}
$$

Taking $c_{2}=\exp L \xi$,we get

$$
\begin{aligned}
H\left(x\left(., x_{0}\right), \hat{0}\right) & =\sup _{0 \leq t \leq \xi} H_{d}\left(x\left(t, x_{0}\right), \hat{0}\right) \\
& \leq c c_{2}\left(H_{d}\left(x_{0}, \hat{0}\right)+N+M+\chi\right) .
\end{aligned}
$$

We consider the following semilinear fuzzy impulsive differential equations with nonlocal conditions:

$$
\begin{aligned}
x(t)= & S(t) x_{0}+S(t) g\left(t_{1}, \ldots, t_{p}, x(.)\right)+\int_{0}^{t} S(t-s) f(s, x(s)) d s \\
& +\sum_{0<t<t_{k}} S\left(t-t_{k}\right) I_{k}\left(x\left(t_{k}\right)\right), \\
x_{n}(t)= & S(t) x_{n, 0}+S(t) g_{n}\left(t_{1}, \ldots, t_{p}, x_{n}(.)\right)+\int_{0}^{t} S(t-s) f_{n}\left(s, x_{n}(s)\right) d s \\
& +\sum_{0<t<t_{k}} S\left(t-t_{k}\right) I_{k}\left(x_{n}\left(t_{k}\right)\right),
\end{aligned}
$$

where $n \geq 1$.If the above mentioned equations satisfies the conditions of Theorem 1 , then they have unique solutions $x(t)$ and $x_{n}(t)$, $t \in[0, \xi]$ respectively.

Theorem 3. Suppose that $f, g$ are the same as mentioned in Theorem 1. If $H_{d}\left(x_{n, 0}, x_{0}\right) \rightarrow 0$,

$$
H_{d}\left(g_{n}\left(t_{1}, \ldots, t_{p}, x(.)\right), g\left(t_{1}, \ldots, t_{p}, x(.)\right)\right) \rightarrow 0
$$


and

$$
\sup _{0 \leq t \leq \xi} H_{d}\left(f_{n}(t, y), f(t, y)\right) \rightarrow 0 \text { as } n \rightarrow \infty
$$

for each $y \in L_{2}$ then

$$
\sup _{0 \leq t \leq \xi} H_{d}\left(x_{n}(t), x(t)\right) \rightarrow 0 \text { as } n \rightarrow \infty .
$$

Proof. For any $0 \leq t \leq \xi$, we have

$$
\begin{aligned}
& H_{d}\left(x_{n}(t), x(t)\right) \\
& \leq c H_{d}\left(x_{n, 0}, x_{0}\right)+c H_{d}\left(g_{n}\left(t_{1}, \ldots, t_{p}, x_{n}(.)\right), g\left(t_{1}, \ldots, t_{p}, x(.)\right)\right) \\
& +c \int_{0}^{t} H_{d}\left(f_{n}\left(s, x_{n}(s)\right), f(s, x(s))\right) d s \\
& +c H_{d}\left(I_{k}\left(x_{n}\left(t_{k}\right)\right), I_{k}\left(x\left(t_{k}\right)\right)\right) \\
& \leq c H_{d}\left(x_{n, 0}, x_{0}\right)+c H_{d}\left(g_{n}\left(t_{1}, \ldots, t_{p}, x_{n}(.)\right), g_{n}\left(t_{1}, \ldots, t_{p}, x(.)\right)\right) \\
& +c H_{d}\left(g_{n}\left(t_{1}, \ldots, t_{p}, x(.)\right), g\left(t_{1}, \ldots, t_{p}, x(.)\right)\right) \\
& +\int_{0}^{t} c H_{d}\left(f_{n}\left(s, x_{n}(s)\right), f_{n}(s, x(s))\right) d s \\
& +\int_{0}^{t} c H_{d}\left(f_{n}(s, x(s)), f(s, x(s))\right) d s+c \beta_{k} \\
& \leq c H_{d}\left(x_{n, 0}, x_{0}\right)+c K H_{d}\left(x_{n}(.), x(.)\right) \\
& +c H_{d}\left(g_{n}\left(t_{1}, \ldots, t_{p}, x(.)\right), g\left(t_{1}, \ldots, t_{p}, x(.)\right)\right) \\
& +c L \int_{0}^{t} H_{d}\left(x_{n}(s), x(s)\right) d s+\int_{0}^{t} c H_{d}\left(f_{n}(s, x(s)), f(s, x(s))\right) d s+c \beta_{k} .
\end{aligned}
$$

From Gronwall's inequality,we get

$$
\begin{aligned}
H_{d}\left(x_{n}(t), x(t)\right) \leq & c\left[H_{d}\left(x_{n, 0}, x_{0}\right)+K H_{d}\left(x_{n}(.), x(.)\right)\right. \\
& +H_{d}\left(g_{n}\left(t_{1}, \ldots, t_{p}, x(.)\right), g\left(t_{1}, \ldots, t_{p}, x(.)\right)\right) \\
& \left.+\int_{0}^{t} H_{d}\left(f_{n}(s, x(s)), f(s, x(s))\right) d s+\beta_{k}\right] \exp L t .
\end{aligned}
$$

That is,

$$
(1-c K \exp L \xi) \sup _{0 \leq t \leq \xi} H_{d}\left(x_{n}(t), x(t)\right)
$$




$$
\begin{aligned}
\leq & c\left[H_{d}\left(x_{n, 0}, x_{0}\right)+H_{d}\left(g_{n}\left(t_{1}, \ldots, t_{p}, x(.)\right), g\left(t_{1}, \ldots, t_{p}, x(.)\right)\right)\right. \\
& \left.+\sup _{0 \leq t \leq \xi} \int_{0}^{t} H_{d}\left(f_{n}(s, x(s)), f(s, x(s))\right) d s+\beta_{k}\right] \exp L \xi
\end{aligned}
$$

And

$$
\begin{aligned}
& H_{d}\left(f_{n}(s, x(s)), f(s, x(s))\right) \\
& \leq \quad H_{d}\left(f_{n}(s, x(s)), f_{n}(s, \hat{0})\right)+H_{d}\left(f_{n}(s, \hat{0}), f(s, \hat{0})\right) \\
& \quad+H_{d}(f(s, \hat{0}), f(s, x(s))) \\
& \leq 2 L H_{d}(x(s), \hat{0})+\sup _{0 \leq s \leq \xi} H_{d}\left(f_{n}(s, \hat{0}), f(s, \hat{0})\right) \\
& \leq 2 L c_{2}\left(H_{d}\left(x_{0}, \hat{0}\right)+N+M+\chi\right)+1
\end{aligned}
$$

as soon as $n$ is large enough,where we used (ii) of the Theorem (2). Since $I_{k}$ is a bounded function,we know that the hypothesis (H5) holds.Hence,by using the dominated convergence theorem in Eq.(4),we obtain the conclusion of the theorem.

\section{References}

[1] D. D. Bainov and P. S. Simeonov, Impulsive Differential Equations: Periodic Solutions and Applications, Longman Scientific and Technical Group, England, (1993).

[2] M. Benchohra, J. J. Nieto and A. Ouahab, Fuzzy solutions for impulsive Differential equations, Communications in Applied Analysis, 11(2007), 379-394.

[3] L. Byszewski, Theorems about the Existence and uniqueness of solutions of semilinear evoultion nonlocal Cauchy problem, J. Math. Anal. Appl, 162(1991), 494-505.

[4] Y. Feng, Mean square integral and differential of fuzzy stochastic processes, Fuzzy Sets and Systems, 102, (1999), 271-280.

[5] Y. Feng, Fuzzy stochastic differential systems, Fuzzy Sets and systems, 115, (2000), 351-363.

[6] J. U. Jeong, Fuzzy differential equations with nonlocal condition, J. Appl. Math. \&Computing, 17, No. 1-2-3(2005), 509-517. 
[7] O. Kaleva, Fuzzy differential equations, Fuzzy sets and Systems, 24(1987), $301-317$.

[8] V. Lakshmikantham, R. N. Mohapatra, Theory of Fuzzy Differential Equations and Inclusions, Taylor \& Francis, London, New York, (2003).

[9] V. Lakshmikantham and F. A. McRae, Basic results for fuzzy impulsive differential equations, Mathematical Inequalities and Applications, 4(2)(2001), 239-246.

[10] V. Lakshmikantham, D. D. Bainov and P. S. Simeonov, Theory of Impulsive Differential Equations, World Scientific, Singapore, (1989).

[11] Heng-you Lan and J. J. Nieto, On initial value problems for First order implicit impulsive fuzzy differential equations, Dynamic system and applications, 18(2009), 677-686

[12] M. L. Puri and D. A. Ralescu, Fuzzy random variables, Fuzzy random variables, J. Math. Anal. Appl. , 114(1986), 409-422.

[13] R. Ramesh and S. Vengataasalam, Existence of solutions of fuzzy impulsive delay integrodifferential equations with nonlocal condition, Far East Journal of Mathematical Sciences, 86, No. 1 (2014), 37-60.

[14] A. M. Samoilenko and N. A. Perestyuk, Impulsive Differential Equations, World Scientific, Singapore, (1995).

[15] S. Seikkala, On the fuzzy initial value problem, Fuzzy sets and Systems, 24(1987), 319-333.

[16] T. T. Tung, On the fuzzy control stochastic differential systems, Control and Cybernetics, 42, (2013), 505-525.

[17] C. Wu, S. J. Song and E. Lee, Appoximate solutions, Existence and uniqueness of the Cauchy problem of fuzzy differential equations, Journal of Mathematical Analysis and Appications, 202, (1996), 629-644.

[18] L. A. Zadeh, Fuzzy sets, Informations and control, 8, No. 3 (1965), pp. $338-353$. 